\title{
QUALIDADE DE VIDA DE ADULTOS COM CÂNCER COLORRETAL COM E SEM OSTOMIA
}

\author{
Adriana de Paula Congro Michelone \\ Vera Lúcia Conceição Gouveia Santos ${ }^{2}$
}

Michelone APC, Santos VLCG. Qualidade de vida de adultos com câncer colorretal com e sem ostomia. Rev Latino-am Enfermagem 2004 novembro-dezembro; 12(6):875-83.

O objetivo deste estudo foi analisar e comparar a qualidade de vida (QV) dos doentes com câncer colorretal atendidos pelo SUS na XIV DIR-SP, conforme ausência e presença de estoma. Trata-se de estudo descritivo e exploratório, com abordagem quantitativa onde se utilizou a escala WHOQOL-bref para avaliação da QV. A casuística foi composta por 110 pessoas, totalizando 48 sobreviventes que compuseram dois grupos conforme ausência ou presença do estoma. Os resultados evidenciaram escores médios menores em todos os domínios para as pessoas ostomizadas, porém sem diferenças significativas na comparação com o grupo de pessoas sem ostomia. Foram constatadas diferenças estatisticamente significantes para as variáveis religião e retorno ao trabalho independente do grupo. O estudo contribuiu para o melhor delineamento acerca da QV entre pessoas operadas por câncer colorretal com e sem ostomia.

DESCRITORES: qualidade de vida; enfermagem oncológica; neoplasias colorretais

\section{QUALITY OF LIFE OF CANCER' PATIENTS WITH AND WITHOUT AN OSTOMY}

This study aimed to analyze and compare the quality of life of patients with colorectal cancer who were attended by the Single Health System in São Paulo. A descriptive and transversal study was carried out from a quantitative approach. Data were collected through the World Health Organization Quality of Life-bref scale (WHOQOL-bref). 110 patients were observed, resulting in a population of 48 patients, who were divided into 2 groups: with and without ostomy. No differences in the average scores for the four domains were found between the groups. Statistical differences were found in the variables of religion and return to work independent from the group. This study was important to get a better understanding of quality of life in cancer patients with and without ostomy.

DESCRIPTORS: quality of life; cancer nursing; colorectal neoplasms

\section{CALIDAD DE VIDA EN ADULTOS CON CÁNCER COLORECTAL CON Y SIN OSTOMÍA}

La finalidad del trabajo fue analizar y comparar la calidad de vida (CV) en enfermos con cáncer colorectal que reciben terapia de SUS en el XIV DIR-SP. Fue efectuado un estudio descriptivo y transversal con aproximación cuantitativa. La escala WHOQOL-bref fue utilizada para evaluar la CV. La casuística fue compuesta de 110 personas, siendo 48 supervivientes que hicieron parte de dos grupos, de acuerdo con la presencia o falta del estoma. Los resultados fueron de puntuaciones medias menores para las personas con ostomía. Todavía no ocurrieron mayores diferencias con relación con el grupo de personas sin ostomía. Fueron encontradas diferencias importantes de acuerdo con la creencia y regresión al trabajo, sin relación al grupo. El estudio ayudó en el progreso para un mejor dibujo respecto a la CV entre personas con intervención quirúrgica debido al cáncer colorectal con y sin ostomía.

DESCRIPTORES: calidad de vida; enfermería oncológica; neoplasmas colorectales

\footnotetext{
${ }^{1}$ Enfermeira, Mestre em Enfermagem pela Escola de Enfermagem da Universidade de São Paulo, Docente da Faculdade de Medicina de Marília, e-mail: adrianap@famema.br; ${ }^{2}$ Enfermeira Estomaterapeuta, Doutor em Enfermagem, Docente da Escola de Enfermagem da Universidade de São Paulo, Orientador, e-mail: veras@usp.br
} 
INTRODUÇÃO

A presença do câncer altera, indubitavelmente, todos os aspectos da vida do indivíduo e pode acarretar profundas alterações no modo de viver habitual, conforme o comprometimento da capacidade e habilidade para execução de atividades de rotina. As alterações da integridade físico-emocional por desconforto, dor, desfiguração, dependência e perda da auto-estima são relatadas por esses doentes que percebem a qualidade de suas vidas profundamente alterada, num curto período de tempo.

A própria palavra "câncer", inclui um simbolismo de ameaça à vida e de associação com a morte e o morrer ${ }^{(1)}$. Nesse aspecto, a pessoa experimenta certos tipos de pavor ao ser acometida por uma "doença mistério" e "moralmente contagiosa", evitando sequer pronunciar o nome da doença ${ }^{(2)}$.

Parece fundamental, portanto, que os enfermeiros e demais elementos da equipe de saúde, envolvidos com essa clientela, tenham visão mais ampliada sobre os sentimentos aflorados frente à doença, às suas seqüelas e aos resultados alcançados no processo reabilitatório. Impõe-se elaborar um plano individualizado de atenção fundamentado no respeito aos valores e crenças do indivíduo que corresponda, de forma mais abrangente, às expectativas e necessidades da pessoa com câncer, contribuindo para a necessária tomada de decisão em direção à melhoria da qualidade de vida, e não apenas ao prolongamento da sobrevida. Na promoção da autonomia do cliente, não cabe à equipe de saúde determinar o que é melhor para ele - o que seria considerado paternalismo - mas instrumentalizá-lo, através do processo educativo, para que ele mesmo possa tomar decisões seguras, com base em seus próprios valores ${ }^{(3)}$.

Como parte das responsabilidades do enfermeiro, essa é uma tarefa muitas vezes difícil que exige conhecimentos e habilidades compromissadas com a humanização do tratamento.

Neste estudo enfoca-se, especificamente, a área da oncologia colorretal na qual, além do estigma do câncer e de sua localização corporal, o indivíduo tem suas preocupações agravadas pelas possibilidades terapêuticas, dentre as quais, o desvio do trânsito intestinal mediante a realização de um estoma.

A pessoa ostomizada, além de sobreviver ao câncer, passa a assumir outras incumbências em presença de tal derivação. A literatura mostra que os indivíduos ostomizados enfrentam várias perdas que podem ser reais ou simbólicas. A perda do controle da eliminação de fezes e gases, condição mandatória para a vida em sociedade, pode acarretar o isolamento psicológico e social, baseado em sentimentos negativos que permeiam as relações interpessoais. Essas pessoas deparam-se com a mutilação de sua imagem corporal e auto-estima, com sentimentos de repugnância de si mesmas, de desprestígio diante da sociedade e de não serem capazes de enfrentar tal situação ${ }^{(2,4-5)}$.

A complexidade e a extensão da problemática inerente à vivência da cronicidade da doença e/ou da seqüela, aqui especialmente representada pelo câncer e pela ostomia, têm levado vários autores a desenvolverem estudos com o objetivo de analisar o impacto dessas condições sobre a qualidade de vida, em diferentes aspectos.

Considerando-se as dificuldades enfrentadas pelos sobreviventes de câncer, sendo ostomizados ou não, ao submergir numa ou duas situações crônicas, câncer e ostomia, que certamente proporcionam drásticas mudanças no estilo de vida, sobrepõe-se, como foco central deste estudo, o grande questionamento: como todo esse processo influencia a qualidade de suas vidas?

Qualidade de vida

Qualidade de vida é uma das mais interdisciplinares terminologias da atualidade ${ }^{(6)}$, sendo utilizada em vários contextos de pesquisa, servindo como elo entre várias áreas especializadas do conhecimento como a sociologia, medicina, enfermagem, psicologia, economia, geografia, história social e filosofia.

Para diversos autores, a qualidade de vida tem componentes subjetivos e objetivos. $\mathrm{O}$ aspecto subjetivo é essencial porque o senso de satisfação pessoal é intrínseco à qualidade de vida. Entretanto, o componente objetivo é também necessário, pois pessoas vivendo em situações de pobreza e miséria podem sentir-se satisfeitas com sua vida, enquanto que outras pessoas enfrentando condições adversas de risco à saúde podem avaliar sua qualidade de vida pior que a desejada.

Ao considerar-se que qualidade de vida é um valor subjetivo, a mesma só pode ser avaliada pela própria pessoa cuja qualidade de vida está sendo $\operatorname{medida}^{(7)}$, contrariamente aos primeiros estudos sobre o tema.

O World Health Organization Quality of Life Group (WHOQOL Group) grupo da Organização Mundial de 
Saúde (OMS) que estuda qualidade de vida baseado em três aspectos referentes ao construto qualidade de vida, quais sejam, subjetividade, multidimensionalidade e presença de dimensões positivas e negativas, define-a como "a percepção do indivíduo de sua posição na vida no contexto da cultura e sistema de valores nos quais ele vive e em relação aos seus objetivos, expectativas, padrões e preocupações" "(7).

A qualidade de vida passa ao âmbito da saúde quando se considera que a doença e as intervenções realizadas pelos profissionais influenciam tanto sobre a quantidade (sobrevivência) como sobre a qualidade da vida do indivíduo ${ }^{(8)}$. No contexto da oncologia, a qualidade de vida é definida como a percepção subjetiva do indivíduo em relação à sua incapacidade e à satisfação com seu nível atual de funcionamento, fazendo com que a pessoa considere que esteja bem ou não, comparativamente ao que percebe como possível ou ideal ${ }^{(9)}$.

A problemática do adulto sobrevivente ao câncer colorretal, certamente exacerbada pela presença de um componente estigmatizante e mutilante como o estoma, aqui colocada em breve revisão de literatura, associada à nossa prática profissional, tanto assistencial quanto de ensino, tem sido fonte de estímulo para aprofundar o conhecimento sobre o universo dessa clientela, constituindo-se como objetivos do estudo:

- analisar e comparar a qualidade de vida dos sobreviventes de câncer colorretal, com e sem ostomia, atendidos pelo Sistema Único de Saúde (SUS) na XIV Divisão Regional da Secretaria de Saúde do Estado de São Paulo;

- verificar as associações estatísticas existentes entre os escores de qualidade de vida e as características demográficas e clínicas entre os dois grupos.

\section{CASUÍSTICA E MÉTODOS}

Trata-se de estudo descritivo e exploratório, de corte transversal, com abordagem quantitativa, no qual se testa a correlação, desenvolvido na XIV Divisão Regional da Secretaria de Saúde do Estado de São Paulo (XIV DIR$\mathrm{SP})$. Essa região é composta por 35 municípios e a principal cidade da região é Marília, onde se localiza a maioria dos hospitais com possibilidade de diagnóstico e tratamento oncológico para os usuários do SUS. Cumpre ressaltar que o Serviço de Anatomia Patológica da Faculdade de Medicina de Marília (FAMEMA) é o serviço credenciado pela DIR-XIV para o diagnóstico anatomopatológico de fragmentos de tecidos e peças cirúrgicas dos usuários do SUS da região.

Inicialmente, o projeto foi submetido à Comissão de Ética de Pesquisa em Seres Humanos da Faculdade de Medicina de Marília e, somente após sua aprovação, deu-se início ao rastreamento da população envolvida e, posteriormente, à coleta de dados propriamente dita.

A população foi constituída de todos os sobreviventes de câncer colorretal atendidos pelo SUS, no período de 1995 a 2000, e residentes na XIV DIR-SP. Para o estabelecimento dessa casuística, procedeu-se a dois rastreamentos. O primeiro foi realizado através dos arquivos do Serviço de Anatomia Patológica da FAMEMA à procura de exames anatomopatológicos de cólon e reto, realizados no período de 01 de janeiro de 1995 a 30 de junho de 2000, sendo identificados 439 exames, dos quais 145 eram positivos para neoplasia colorretal, do tipo adenocarcinoma. Estabeleceu-se o período de cinco anos para esse rastreamento devido aos índices de sobrevida para esse tipo de câncer.

Após identificação das datas dos exames e respectivos usuários, foram confirmadas seis instituições que prestaram atendimento a essa clientela. A partir daí, realizou-se contato telefônico com todos os hospitais envolvidos, visando identificar os nomes dos diretores clínicos para envio de ofício solicitando o endereço residencial e número de telefone dos pacientes.

Após a obtenção dos 145 endereços residenciais fornecidos pelos hospitais, realizou-se o segundo rastreamento, dessa vez junto à clientela, através de contatos telefônicos ou visita domiciliária para confirmação do endereço residencial, explicação dos objetivos da pesquisa e agendamento de visita domiciliária para entrevista, desde que os participantes atendessem aos seguintes critérios de inclusão: ter idade igual ou superior a 18 anos de idade, residir nas cidades pertencentes à DIR-XIV, possuir condições físicas, capacidade de compreensão e verbalização adequadas para responder à entrevista e aceitar participar do estudo.

Nesses contatos, foram excluídos 46 pessoas que estavam atualmente residindo em outras cidades não pertencentes à DIR-XIV SP, restando, portanto, 99 pessoas.

Ao término do segundo rastreamento, excluídos os 49 não sobreviventes, além de duas pessoas que não apresentavam condições físicas para entrevista, obtiveramse 48 indivíduos, dos quais 31 (64,6\%) sem ostomia e 17 $(35,4 \%)$ com ostomia, que passaram a compor os dois 
grupos, para efeito de análise e discussão dos resultados.

Os dados foram coletados por meio de entrevistas no domicílio do entrevistado, em situação de privacidade, utilizando-se como referência para as respostas as últimas duas semanas. Embora, na maioria das vezes, houvesse algum membro da família presente no recinto, o mesmo era orientado a não intervir. Antes de iniciar as entrevistas, todos eram novamente informados dos objetivos da pesquisa, do sigilo das informações obtidas, bem como do modo de aplicação e do destino dos dados, sendo solicitados a assinarem o termo de consentimento.

Para a coleta de dados, empregou-se um instrumento que constou de duas partes. A primeira destinada à caracterização dos dados sociodemográficos (sexo, idade, escolaridade, situação conjugal, religião, perfil ocupacional e retorno ao trabalho) e clínicos (presença ou ausência de ostomia, tratamento adjuvante, data da cirurgia, e outras doenças associadas) e a segunda parte contendo a Escala WHOQOL -bref, versão em português.

\section{Escalas WHOQOL}

A ausência de um instrumento para avaliação da qualidade de vida da população ao redor do mundo motivou a Organização Mundial de Saúde (OMS) à criação de um Grupo de Estudos sobre Qualidade de Vida (WHOQOL Group), com a finalidade de estudar o conceito e desenvolver instrumentos de medida, numa perspectiva transcultural. O método WHOQOL foi desenvolvido de forma colaborativa e simultânea em vários centros, incluindo países com diferentes níveis de industrialização, disponibilidade de serviços de saúde, importância da família e religiões dominantes, entre outros, além de utilizar uma entrada de dados interativa entre os pesquisadores, em cada estágio de seu desenvolvimento, permitindo constante influência no processo ${ }^{(7)}$. O instrumento inicialmente elaborado, WHOQOL-100, é composto por 100 perguntas que se referem a 6 domínios: físico, psicológico, nível de independência, relações sociais, meio-ambiente e espiritualidade. Embora tratando-se de instrumento bastante complexo e amplo, a necessidade de versões mais curtas que demandassem pouco tempo para o seu preenchimento, levou o mesmo grupo ao desenvolvimento da forma abreviada do WHOQOL-100, ou seja, o WHOQOL-bref que manteve a adequação das propriedades psicométricas do instrumento original ${ }^{(10)}$.

O WHOQOL-bref é composto por 26 itens que representam as facetas, as quais, por sua vez, referem- se a 4 domínios: físico, psicológico, relações sociais e meio ambiente. Os domínios físico e psicológico incluíram, respectivamente, os domínios nível de independência e espiritualidade da versão completa original. Os domínios são constituídos pelas mesmas 24 facetas do formato original, avaliadas por questões únicas, além de duas perguntas de avaliação geral sobre qualidade de vida.

As respostas para todas as questões do WHOQOL-brefsão obtidas através de uma escala do tipo Likert de cinco pontos, na qual a pontuação pode variar de 1 a 5 , além de duas questões sobre qualidade de vida geral calculadas em conjunto para gerar um único escore independente dos escores dos domínios, denominada overall ou "qualidade de vida geral".

Para o cálculo dos escores dos domínios, os índices das facetas componentes resumem os domínios aos quais pertencem. Tanto os domínios como a qualidade de vida geral são medidos em direção positiva, ou seja, escores mais altos denotam melhor qualidade de vida. Para esse cálculo, utilizou-se a sintaxe oferecida pelo WHOQOL Group para ser usada no programa de software SPSS (Statistical Package for Social Science). Com ele o programa pôde checar, recodificar e estabelecer os escores dos domínios e das questões de qualidade de vida geral.

Cumpre enfatizar que tanto o WHOQOL-brefcomo o WHOQOL-100 não permitem o estabelecimento de um escore total único. Ambos instrumentos foram desenvolvidos a partir da premissa de que qualidade de vida é um construto multidimensional, havendo, portanto, maior coerência ao considerar-se cada domínio e respectivo escore individualmente ${ }^{(7,10)}$.

Para atender os objetivos propostos foi realizada análise estatística descritiva dos dados sociodemográficos e clínicos e do WHOQOL-bref, através do cálculo da média, desvio padrão e proporções e análise inferencial por meio dos seguintes procedimentos estatísticos: intervalo de confiança de 95\% (estimativa do valor médio real das variáveis quantitativas e porcentagens reais de alguns eventos de interesse do estudo), teste $t$-student (comparação entre as médias de variáveis de duas populações independentes), análise de variância (comparação entre as médias de variáveis de mais de duas populações independentes com distribuição normal), coeficiente de correlação linear de Pearson (avaliação das correlações entre variáveis numéricas contínuas) e análise de regressão linear múltipla (avaliação das variáveis que 
permaneceram estatística e independentemente associadas com as questões que avaliam QV geral). Além desses, utilizou-se ainda o coeficiente Alpha de Cronbach e testes de correlação e comparação (coeficiente de correlação de Pearson, correlação linear e regressão linear múltipla) para avaliação das propriedades psicométricas do WHOQOL-brefpara este estudo. Em todas as análises, o p-value inferior a 0,05 foi considerado estatisticamente significante.

\section{RESULTADOS}

Para utilização do WHOQOL-bref, junto à população, o mesmo foi submetido à avaliação de algumas de suas propriedades psicométricas como a confiabilidade - através de consistência interna - e validade de construto e de critério concorrente, considerando o total de 48 entrevistados.

A consistência interna do instrumento foi verificada através do coeficiente de fidedignidade Alpha de Cronbach quanto aos domínios $(0,73)$, facetas $(0,91)$ e cada domínio separadamente - físico $(0,88)$, psicológico $(0,74)$, relações sociais $(0,73)$ e meio ambiente $(0,60)$ - resultados considerados satisfatórios para atestar a consistência interna do instrumento para este estudo.

A validade de construto pode ser avaliada por meio das correlações entre os escores dos diferentes domínios, verificando-se valores satisfatórios e correlações moderadas e estatisticamente significantes entre os domínios físico e psicológico $(r=0,54)$ e domínios físico e relações sociais $(r=0,56)$. Já o domínio meio ambiente apresentou correlações fracas e estatisticamente significantes com domínio físico $(r=0,49)$ e domínio psicológico $(r=0,29)$.

A validade de critério concorrente foi avaliada através da análise de correlação entre as questões que medem QV geral e os quatro domínios do WHOQOL-bref. Verificou-se que o domínio físico está fortemente correlacionado, positivamente e significativamente do ponto de vista estatístico, com a qualidade de vida geral $(r=0,70)$, enquanto que os domínios psicológico $(r=0,55)$ e relações sociais $(r=0,57)$ estão correlacionados de forma moderada e estatisticamente significantes com a QV geral. O domínio meio ambiente apresentou, novamente, o menor valor de correlação $(r=0,20)$ e sem significância estatística.

A validade de critério concorrente foi também avaliada pela regressão linear múltipla entre os diversos domínios em relação à $Q V$ geral, mostrando que apenas - domínio físico permaneceu estatística e independentemente associado às questões que avaliam QV geral, explicando 60\% de variância.

Quanto às características sociodemográficas da população, em ambos os grupos, houve predomínio de pessoas do sexo masculino (56,2\%), baixa escolaridade $(85,4 \%)$, de religião católica $(81,2 \%)$ e exercendo atividades remuneradas (45,8\%).

No grupo de pessoas sem ostomia a média de idade foi 60,9 anos (DP=12,07), situação conjugal com companheiro $(64,5 \%)$, e que retornaram ao trabalho após o tratamento $(74,2 \%)$. No grupo dos ostomizados a média de idade foi de 66,5 anos ( $D P=15,97$ ), vivendo sem companheiro (52,9\%), e que não retornaram ao trabalho após o tratamento $(70,6 \%)$.

Diferenças estatisticamente significantes foram constatadas entre os grupos em relação à religião $(p=0,03)$ e em relação ao retorno ao trabalho $(p=0,003)$.

Quanto às variáveis clínicas, em ambos os grupos, a maioria dos entrevistados não estava sendo submetida a qualquer tratamento adjuvante (quimioterapia ou radioterapia) nos 15 dias anteriores à entrevista $(79,1 \%) \mathrm{e}$ as médias de tempo após a cirurgia foram de 32,6 meses ( $D P=21,8)$ no grupo sem ostomia e de 20,2 meses ( $D P=20)$ no grupo de ostomizados.

Análise da qualidade de vida

Os escores médios obtidos nos quatro domínios do WHOQOL-brefe nas questões que medem qualidade de vida geral são apresentados na Tabela 1.

Tabela 1 - Escores médios dos domínios e qualidade de vida geral do WHOQOL-bref, segundo o grupo. DIR XIV SP, novembro 2000

\begin{tabular}{|c|c|c|c|c|c|c|c|c|}
\hline \multicolumn{2}{|c|}{ Grupos } & \multicolumn{3}{|c|}{ Sem ostomia } & \multicolumn{4}{|c|}{ Com ostomia } \\
\hline Domínios & $n$ & Média & DP & IC 95\% & Média & DP & IC95\% & $p$ \\
\hline Físico & 48 & 68,1 & $(23,7)$ & $59,4-76,8$ & 58,6 & $(18,6)$ & $49,0-68,2$ & 0,15 \\
\hline Psicológico & 48 & 68,2 & $(29,5)$ & $57,4-79,1$ & 62,7 & $(17,4)$ & $53,7-71,7$ & 0,48 \\
\hline Relações Sociais & 39 & 66,6 & $(23,6)$ & $57,0-76,2$ & 64,7 & $(21,0)$ & $52,0-77,4$ & 0,80 \\
\hline Meio Ambiente & 48 & $\begin{array}{c}65,3 \\
(77,0)\end{array}$ & $(26,8)$ & $55,4-75,1$ & $\begin{array}{c}60,1 \\
(78,5)\end{array}$ & $(11,5)$ & $54,1-66,0$ & 0,45 \\
\hline QV geral & 48 & 15,4 & $(3,13)$ & $14,3-16,6$ & 15,7 & $(3,38)$ & $14,0-17,5$ & 0,77 \\
\hline
\end{tabular}


Os dados da Tabela 1 mostram escores médios para os domínios que não diferem estatisticamente entre os grupos, embora sejam menores para os pacientes com ostomia.

Cabe ressaltar que, neste estudo, obteve-se ausência de respostas em relação à faceta atividade sexual para 9 entrevistados, o que gerou um total de 39 respondentes no domínio relações sociais, onde tal faceta está inserida.

Quanto ao escore médio de qualidade de vida geral, os valores foram similares entre os grupos, novamente sem diferença estatisticamente significativa. Para melhor compreensão dessa resposta, quando perguntados como consideram sua qualidade de vida, 17 $(54,8 \%)$ pessoas do grupo sem ostomia e $9(52,9 \%)$ pessoas do grupo com ostomia avaliaram a qualidade de suas vidas como "boa", enquanto $6(19,3 \%)$ e $4(23,5 \%)$ pessoas dos grupos sem e com ostomia, respectivamente, como "muito boa", portanto, avaliações bastante positivas, conforme também constatado através dos escores médios dos domínios.

Cabe ressaltar que, neste estudo, obteve-se ausência de respostas em relação à faceta atividade sexual para 9 entrevistados o que gerou um total de 39 respondentes no domínio relações sociais, onde tal faceta está inserida.

A partir dos resultados obtidos, buscou-se identificar as facetas mais influentes para a qualidade de vida em cada um dos domínios do WHOQOL-bref, através da correlação entre cada questão que compõe o domínio e seu escore médio. O reduzido número de componentes de cada grupo impossibilitou a avaliação dessas facetas por grupo.

Nas Tabelas 2, 3, 4 e 5 são apresentados os resultados encontrados para cada domínio.

Tabela 2 - Correlação entre cada questão e o escore médio do Domínio Físico. DIR XIV SP, novembro 2000

\begin{tabular}{clcc}
\hline $\begin{array}{c}\text { № da } \\
\text { questão }\end{array}$ & \multicolumn{1}{c}{ Faceta avaliada } & Correlação & \multicolumn{1}{c}{$\mathrm{p}$} \\
\hline $\mathbf{3}$ & Dor e desconforto & $-\mathbf{0 , 7 5 ^ { * }}$ & $<\mathbf{0 , 0 0 1}$ \\
$\mathbf{4}$ & $\begin{array}{l}\text { Dependência de medicação } \\
\text { ou tratamentos }\end{array}$ & $-0,66^{*}$ & $<0,001$ \\
10 & Energia e fadiga & $0,56^{\star}$ & $<0,001$ \\
$\mathbf{1 5}$ & Mobilidade & $\mathbf{0 , 8 4 ^ { \star }}$ & $<0,001$ \\
16 & Sono e repouso & $0,48^{\star}$ & $<0,001$ \\
$\mathbf{1 7}$ & Atividades da vida & $\mathbf{0 , 7 1 ^ { * }}$ & $<0,001$ \\
& cotidiana & & \\
$\mathbf{1 8}$ & Capacidade de trabalho & $\mathbf{0 , 7 7 ^ { \star }}$ & $<\mathbf{0 , 0 0 1}$ \\
\hline
\end{tabular}

(*) A correlação é estatisticamente significante
Os dados da Tabela 2 revelam que todas as facetas do domínio físico estão correlacionadas com o escore médio obtido em tal domínio, sendo as facetas Mobilidade, Capacidade de trabalho, Dor e desconforto e Atividades da vida cotidiana aquelas que mais influenciaram o escore médio do domínio, nesta ordem.

Tabela 3 - Correlação entre cada questão e o escore médio do Domínio Psicológico. DIR XIV SP, novembro 2000

\begin{tabular}{|c|c|c|c|}
\hline $\begin{array}{l}\text { № da } \\
\text { questão }\end{array}$ & Faceta avaliada & Correlação & $\mathrm{p}$ \\
\hline 5 & Sentimentos positivos & $0,49^{*}$ & $<0,001$ \\
\hline 6 & $\begin{array}{l}\text { Espiritualidade/ religião/ } \\
\text { crenças pessoais }\end{array}$ & $0,77^{*}$ & $<0,001$ \\
\hline 7 & $\begin{array}{l}\text { Pensar, aprender, memória e } \\
\text { concentração }\end{array}$ & 0,25 & 0,07 \\
\hline 11 & Imagem corporal e aparência & $0,40^{*}$ & 0,005 \\
\hline 19 & Auto-estima & $0,41^{*}$ & 0,003 \\
\hline 26 & Sentimentos negativos & $-0,27$ & 0,057 \\
\hline
\end{tabular}

$\left(^{*}\right)$ A correlação é estatisticamente significante

Os dados da Tabela 3 mostram que quatro dentre seis facetas do domínio Psicológico correlacionam-se positiva e significativamente com o seu escore médio, em maior ou menor intensidade, sendo a Espiritualidade/ religião/crenças pessoais a mais influente nesse domínio.

Tabela 4 - Correlação entre cada questão e o escore médio do Domínio Relações Sociais. DIR XIV SP, novembro 2000

\begin{tabular}{clcc}
\hline $\begin{array}{c}\text { № da } \\
\text { questão }\end{array}$ & Faceta avaliada & Correlação & $\mathrm{p}$ \\
\hline 20 & Relações pessoais & $0,86^{*}$ & $<0,001$ \\
21 & Atividade sexual & $0,78^{*}$ & $<0,001$ \\
22 & Suporte (apoio) social & $0,77^{*}$ & $<0,001$ \\
\hline
\end{tabular}

(*) A correlação é estatisticamente significante

Os dados da Tabela 4 indicam que as três facetas que compõem o domínio Relações Sociais estão positiva e fortemente correlacionadas com o escore médio obtido em tal domínio, com significância estatística $(p<0,001)$.

Tabela 5 - Correlação entre cada questão e o escore médio do Domínio Meio Ambiente. DIR XIV SP, novembro 2000

\begin{tabular}{clcc}
\hline $\begin{array}{c}\text { № da } \\
\text { questão }\end{array}$ & \multicolumn{1}{c}{ Faceta avaliada } & Correlação & $\mathrm{p}$ \\
\hline $\mathbf{8}$ & Segurança física e proteção & $\mathbf{0 , 3 9 ^ { * }}$ & $\mathbf{0 , 0 0 6}$ \\
9 & $\begin{array}{l}\text { Ambiente físico (poluição, ruído, } \\
\text { trânsito, clima) }\end{array}$ & 0,25 & 0,07 \\
12 & Recursos financeiros & 0,23 & 0,10 \\
$\mathbf{1 3}$ & $\begin{array}{l}\text { Oportunidades de adquirir novas } \\
\text { informações e habilidades }\end{array}$ & $\mathbf{0 , 5 0 *}$ & $\mathbf{< 0 , 0 0 1}$ \\
$\mathbf{1 4}$ & Participação e oportunidades de & $\mathbf{0 , 4 2 ^ { * }}$ & $\mathbf{< 0 , 0 0 1}$ \\
& recreação e lazer & 0,18 & 0,20 \\
23 & Ambiente no lar & 0,26 & 0,06 \\
24 & Cuidados de saúde e sociais & 0,17 & 0,23 \\
\hline 25 & Transporte & & \\
\hline
\end{tabular}

(*) A correlação é estatisticamente significante 
Os dados da Tabela 5 revelam que esse foi 0 domínio com valores de correlação mais fracos, sendo que apenas três das oito facetas apresentam-se estatística e fracamente correlacionadas com o escore médio obtido no domínio, exatamente aquelas consideradas mais influentes. Desse modo, Oportunidades de adquirir novas informações e habilidades, Participação e oportunidades de recreação e lazer e Segurança física e proteção, nessa ordem decrescente, foram as facetas que mais influenciaram o escore médio do domínio Meio Ambiente.

A seguir, buscou-se comparar as variáveis sociodemográficas e clínicas de ambos os grupos em cada domínio do WHOQOL-bref. Foram identificadas diferenças estatisticamente significantes entre os grupos para a variável religião no domínio Físico $(\mathrm{p}=0,02)$ e para a variável retorno ao trabalho no domínio Relações Sociais $(p=0,04)$. Os resultados mostram que a diferença ocorreu internamente no grupo de pessoas com ostomia em relação à religião, isto é, os católicos ostomizados apresentam escores maiores de qualidade de vida no domínio Físico quando comparados aos indivíduos de outras religiões e ostomizados; e, internamente, no grupo de pessoas não ostomizadas entre as pessoas que voltaram ao trabalho comparativamente àquelas que não retornaram ao trabalho após o tratamento do câncer.

\section{DISCUSSÃo}

Do ponto de vista sociodemográfico, houve predomínio do sexo masculino, o que vai ao encontro de autores que afirmam ser o câncer colorretal mais freqüente em homens ${ }^{(12)}$. Com relação à idade, os resultados são confirmados pela literatura que indica cerca de $70 \%$ dos portadores desse tipo de neoplasia na faixa etária dos 40 aos 70 anos de idade ${ }^{(11)}$.

Outro aspecto demográfico importante é a situação conjugal da clientela. Estudos internacionais com pessoas portadoras de câncer colorretal apontam entre 60 e 95\% de pessoas casadas ${ }^{(11)}$ e alguns estudos nacionais com indivíduos ostomizados indicam que a maior parte vive com companheiros, com percentuais de 61,9 a $87,5 \%{ }^{(12)}$.

Em relação ao retorno ao trabalho os resultados são, em parte, confirmados por autores segundo os quais 79 a $93 \%$ dos sobreviventes de câncer colorretal sem ostomia e 20 a $72 \%$ com ostomias reassumem seu trabalho após o tratamento ${ }^{(13)}$. Outros pesquisadores mencionam que a capacidade de trabalho é afetada entre 20 e 90\% dos indivíduos ostomizados devido à idade e não ao estoma, ou à doença, propriamente dito ${ }^{(14)}$.

Uma vez caracterizada a população portadora de câncer colorretal, passa-se à análise de sua qualidade de vida.

Ao serem avaliados os escores médios de qualidade de vida obtidos nos diversos domínios (Tabela 1), os pacientes não ostomizados apresentaram maiores escores médios para todos os domínios quando comparados àqueles obtidos pelos ostomizados, apesar de não serem constatadas diferenças significativas entre os grupos. Similarmente, num estudo sobre qualidade de vida do mesmo tipo de pacientes, os autores declaram que a presença da colostomia não parece ter afetado significativamente a qualidade de vida dessa clientela ${ }^{(11)}$. Ao se tentar estabelecer comparação entre os índices de qualidade de vida obtidos nesta pesquisa, não se identificou qualquer estudo semelhante relacionado à avaliação de qualidade de vida do mesmo tipo de clientela utilizando-se o WHOQOL-bref. Entretanto, como esse instrumento foi empregado em outros estudos, envolvendo pessoas com outras doenças crônicas, passamos a discutir os resultados encontrados à luz desses trabalhos.

Em estudo com 66 pacientes com depressão, na Argentina, encontrou-se escores médios que variaram de 41,4 a 50,6 (domínio Físico), 33,25 a 43,65 (domínio Psicológico), 38,45 a 46,05 (domínio Relações Sociais) e 46,90 a 56,65 (domínio Meio Ambiente), segundo o grau de depressão severa ou moderada ${ }^{(15)}$. Comparando-se esses resultados com os deste estudo, evidencia-se, que, para todos os domínios, os escores médios de pessoas com depressão severa ou moderada são inferiores aos encontrados para doentes com câncer colorretal, indicando que, embora sejam portadores de uma neoplasia maligna, e mesmo em presença de uma ostomia, essa clientela apresenta escores médios de qualidade de vida superiores em todos os domínios do WHOQOL-bref.

Em relação aos escores de qualidade de vida geral, esse dado não foi mencionado em nenhum outro estudo utilizando o WHOQOL-bref, tendo-se encontrado apenas os resultados de um estudo utilizando o WHOQOL100 na Holanda, com 32 pacientes com artrite reumatóide, 37 pacientes com sarcoidose e 37 indivíduos saudáveis como grupo-controle ${ }^{(16)}$. Esses pesquisadores encontraram escore médio de qualidade de vida geral de 13,6 (DP=2,5) para o grupo com artrite reumatóide, 14,2 
$(\mathrm{DP}=3,7)$ para o grupo com sarcoidose e 16,2 (DP=2,3) para o grupo controle. Neste estudo, ao utilizar-se a mesma variação de 4 a 20, o que corresponderia a 15,4 (DP=3,13) para o grupo sem ostomia e 15,7 ( $\mathrm{DP}=3,38)$ para o grupo com ostomia, verifica-se que o escore médio de qualidade de vida geral é ligeiramente superior entre os entrevistados com câncer quando comparado aos índices obtidos pelos holandeses portadores de artrite reumatóide e sarcoidose, sendo inferior apenas aos indivíduos saudáveis.

Ao se identificar as facetas mais influentes sobre o escore de cada domínio do WHOQOL-bref, constataramse no domínio Físico (Tabela 2) que todas as facetas encontram-se correlacionadas indicando que a possibilidade de mover-se livremente, a satisfação com a capacidade para o trabalho e para as atividades rotineiras contribuem para os melhores índices de qualidade de vida na esfera física, enquanto a presença da dor e desconforto piora a percepção sobre qualidade de vida nesse domínio.

Para o domínio Psicológico (Tabela 3) foi a espiritualidade, religião, crenças pessoais $(r=0,77)$, a faceta que mais contribuiu para os melhores escores de qualidade de vida neste domínio. Demonstrando que ao serem enfrentados - tanto o tipo de doença como a presença do estoma -, através da religiosidade, esses fatores podem contribuir para a melhor qualidade de vida na esfera psicológica.

As facetas relações pessoais $(r=0,86)$, atividade sexual $(r=0,78)$ e suporte social $(r=0,77)$ foram todas determinantes dos melhores escores de qualidade de vida no domínio Relações Sociais (Tabela 4), também revelando a importância do suporte social que se inicia na família, e da sexualidade para o melhor desempenho na área dos relacionamentos pessoais.

O domínio Meio Ambiente mostrou as correlações mais fracas para a determinação das facetas mais influentes no escore médio do domínio (Tabela 5). No estudo argentino anteriormente citado, os autores também referem correlações mais baixas nesse domínio justificando que, embora todos os itens desse domínio compreendam elementos relacionados ao meio ambiente, não necessariamente estão estreitamente relacionados entre $\mathrm{si}^{(16)}$.

Ao identificar, através de testes de comparação, como as variáveis sociodemográficas e clínicas relacionam-se com os diversos domínios da qualidade de vida, verificou-se que a religião, independente da crença, revela sua importância como outra fonte de suporte e enfrentamento, o que também se comprovou como uma das facetas mais influentes no domínio psicológico, quando compreendida como essência da espiritualidade e das crenças pessoais. Também a variável retorno ao trabalho obteve diferença estatisticamente significante no interior do grupo de pessoas sem ostomia, o que parece ser coerente à medida da maior intensidade de relacionamentos proporcionada pelas atividades laborais.

É importante ressaltar que, apesar da prevalência e sobrecarga física e emocional do câncer colorretal sobre os pacientes, suas famílias e sociedade, existem poucas informações acerca da qualidade de vida dessa clientela, embora exista um foco crescente na "qualidade da sobrevivência". Sobre isso, alguns pesquisadores ${ }^{(11)}$ recomendam a utilização de abordagens que possibilitem diferenciar pacientes com remissão da doença daqueles com recidiva, quando se avalia a qualidade de vida de sobreviventes de câncer colorretal.

As pesquisas nessa área possibilitam obter informações que podem ajudar os profissionais de saúde a atentarem para o fortalecimento do vínculo profissional, e também auxiliar os doentes/familiares na tomada de suas decisões, assim, os enfermeiros e toda equipe de saúde devem estar preparados para encorajar os pacientes a falar sobre seus sentimentos, situação que pode ajudálos a aceitar as várias mudanças que atravessam ou irão atravessar.

Apesar da limitada casuística e mesmo da ausência de um grupo controle com pessoas saudáveis, este estudo certamente contribui para a reflexão acerca da qualidade de vida de pessoas com câncer e como a presença de um estoma pode influenciá-la. Com grupos de pessoas bastante semelhantes do ponto de vista metodológico, não foram detectadas importantes diferenças na qualidade de vida de ambos grupos, mas alguns aspectos como trabalho e religião puderam ser melhor discutidos. Além disso, também foram, novamente, ratificadas importantes características da qualidade de vida como subjetividade e multidimensionalidade do construto, ao serem verificados escores bastante positivos de qualidade de vida apesar da presença do câncer e ostomia. Ressalta-se, ainda, que o tema é suficientemente complexo e instigante para permitir ou indicar a realização de estudos mais abrangentes, utilizando a mesma metodologia criteriosamente descrita, além de referendar o uso do WHOQOL-brefem nosso meio e junto à clientela portadora de câncer colorretal com e sem ostomia. 
1. Muthny FA, Koch U, Stump S. Quality of life in oncology patients. Psychother Psychosom 1990; 54:145-60.

2. Trentini M, Silva DMGV, Pacheco MAB, Martins ML. Ajuda: uma fonte de forças na vida das pessoas ostomizadas. Cogitare Enfermagem 1997; 2(1):3-8.

3. Jassak PF, Ryan MP. Ethical issues in clinical research. Semin Oncol Nurs 1989; 5:102-8.

4. Boccardo LM, Nogueira SA, Santos ER, Miyadahira AMK, Santos VLCG. Aspectos da reinserção social do ostomizado. Rev Esc Enfermagem USP 1995; 29:59-71.

5. Oliveira DVD, Nakano TTY. Reinserção social do ostomizado. In: Santos VLCG, Cesaretti IUR. Assistência em Estomaterapia: cuidando do ostomizado. São Paulo: Atheneu; 2001. p. 279-90.

6. Farquhar M. Definitions of quality of life : a taxonomy. J Adv Nurs 1995; 22:502-8.

7. Fleck MPA. O instrumento de avaliação de qualidade de vida da Organização Mundial de Saúde (WHOQOL-100): características e perspectivas. Ci Saúde Coletiva 2000; 5:338.

8. Lara-Muñoz MC, Ponce de León S, Ramón de la Fuente J. Conceptualización y medición de la calidad de vida de pacientes con cáncer. Rev Invest Clin 1995; 47:315-27.

9. Cella DF, Tulsky DS. Measuring the quality of life today: methodological aspects. Oncology (Huntingt) 1990; 4(5):2938.

10. Fleck MPA, Louzada S, Xavier M, Chachamovich E, Vieira G, Santos L, et al. Aplicação da versão em português do instrumento abreviado de avaliação da qualidade de vida "WHOQOL-BREF". Rev Saúde Pública 2000; 34:178-83.

11. Ramsey SD, Andersen MR, Etzioni R, Moinpour C, Peacock S, Potosky A, et al. Quality of life in survivors of colorectal carcinoma. Cancer 2000; 88:1294-303.

12. Bandeira RCF, Wirthmann E, Costa MQ, Kimura M, Chaves EC, Santos VLCG. Qualidade de vida e coping de pessoas portadoras de estomas definitivos. [CD-ROM] In: 4 Congresso Brasileiro de Estomaterapia; 10 Congresso Brasileiro de Enfermagem em Dermatologia; 2001 out 22-25; São Paulo: SOBEST; 2001.

13. Sprangers MAG, Taal BG, Aaronson NK, te Velde A. Quality of life in colorectal cancer: stoma vs. Nonstoma patients. Dis Colon Rectum 1995; 38:361-9.

14. Rubin GP, Devlin B. The quality of life with a stoma. $\mathrm{Br} \mathrm{J}$ Hosp Med 1987; 38:300-3,6.

15. Bonicatto S, Zaratiegui R, Lorenzo L, Pesina P. Evaluación de calidade de vida en pacientes com depresión mayor: predictibilidad de los niveles de severidad. Acta Psiquiatr Psicol Am Lat 2000; 46:318-24.

16. Wirnsberger RM, De Vries J, Jansen TLTA, Van Heck GL, Wouters EFM, Drent M. Impairment of quality of life: rheumatoid artritis versus sarcoidosis. Neth J Med 1999; 54:86-95. 